05,10

\title{
Динамика магнитных скирмионов в наноточках
}

\author{
(C) 3.В. Гареева ${ }^{1,2}$, К.Ю. Гуслиенко ${ }^{3,4}$ \\ ${ }^{1}$ Институт физики молекул и кристаллов - обособленное структурное подразделение Федерального государственного \\ бюджетного научного учреждения Уфимского фредерального исследовательского центра Российской академии наук, \\ Уфа, Россия \\ ${ }^{2}$ Башкирский государственный университет, \\ Уфра, Россия \\ ${ }^{3}$ Depto. Física de Materiales, Facultad de Química, Universidad del País Vasco, UPV/EHU, \\ 20018 San Sebastián, Spain \\ ${ }^{4}$ IKERBASQUE, the Basque Foundation for Science, \\ 48013 Bilbao, Spain \\ E-mail: gzv@anrb.ru
}

Исследованы магнитные скирмионы Блоха и Нееля в системах ограниченной геометрии (наноточках, линейном массиве наноточек). Рассчитаны спектры низкочастотных и высокочастотных мод возбуждений скирмионного состояния. Показано, что асимметрия скирмионного спектра, а именно, характерное различие частот азимутальных мод скирмионов, вращающихся по и против часовой стрелки, связано с асимметрией профилей намагниченности высокочастотных спиновых волн, распространяющихся на фоне скирмионного состояния в наноточке. В низкочастотном спектре выделяется единственная гиротропная мода, локализованная в окрестности центра наноточки. Значение гиротропной частоты зависит от материальных параметров наноточки и размера скирмиона. Собственная частота гиротропной моды изолированного скирмиона в наноточке в ультратонких пленках $(L \sim 1 \mathrm{~nm})$ не зависит от внутренней структуры скирмиона и является одинаковой для скирмионов блоховского и неелевского типов. Взаимодействие скирмионов, в частности, в линейной цепочке наноточек с основным скирмионным состоянием, приводит к различиям низкочастотных спектров. Структура скирмиона (Блоха или Нееля) проявляется в сдвиге дисперсионных кривых и различии частот ферромагнитного резонанса системы взаимодействующих скирмионов.

Работа 3.В.Г. поддержана Российским фондом фундаментальных исследований (грант № 16-02-00336 А). Работа К.Ю.Г. поддержана IKERBASQUE (the Basque Foundation for Science), the Spanish MINECO grant FIS2016-78591-C3-3-R and the European Union's Horizon 2020 research and innovation program under the Marie Skłodowska-Curie grant N 644348.

DOI: $10.21883 /$ FTT.2018.06.45988.23M

\section{1. Введение}

Магнитные вихри и скирмионы, являясь устойчивыми наноразмерными объектами, привлекают активное внимание исследователей, что связано с перспективами развития спинтроники, устройств сверхплотного хранения информации, магнитной памяти нового поколения, в которых в качестве активных функциональных элементов могут выступать скирмионы. Скирмионы, разновидность 2D магнитных солитонов, являются микромагнитными объектами с нетривиальной топологией и представляют несомненный интерес для фундаментальных исследований.

Скирмионные состояния - это локализованные неоднородные распределения намагниченности с выраженной $m_{z}$ компонентой, перпендикулярной к плоскости системы, в которой реализуются скирмионы. Скирмионы имеют ненулевой топологический заряд $(N=1)$ и характеризуются сильной локализацией (размеры магнитных скирмионов, которые были получены на сегодняшний день, составляют 50-100 nm). Для реализации скирмионных состояний требуются определенные условия. Скирмионные гексагональные решетки возникают в кристаллах В20 (типа $\mathrm{MnSi}$ ) с нарушенной простран- ственной инверсией [1-3], а индивидуальные скирмионы генерируются при приложении магнитного поля и действии электрического тока [4-6] в ультратонких пленках и наноточках под действием взаимодействия Дзялошинского-Мория.

В настоящее время ведется активный поиск методов получения устойчивых высокотемпературных скирмионов, что достигается в материалах с сильной спинорбитальной связью - в ультратонких пленках $\mathrm{Co} / \mathrm{Pt}$, $\mathrm{Pt} / \mathrm{Co} / \mathrm{Ir}$, в которых взаимодействие ДзялошинскогоМория, возникающее на интерфейсе, стабилизирует скирмионные состояния [4-6]; в искусственных средах: структурированных пленках (patterned films), сочетающих магнитомягкие и магнитожесткие слои [7], а также в периодических массивах наноточек с устойчивой скирмионной конфигурацией в основном состоянии [8-10].

Динамика скирмионов представляет большой интерес с точки зрения перспективы практических приложений. Топологический и скирмионный эффекты Холла, связанные с ненулевым топологическим зарядом; зонная структура спектра скирмионных магнонных кристаллов; возможность динамического управления скирмионными состояниями электрическими токами малых плотностей в энергосберегающем режиме открывают широкие пер- 
спективы использования скирмионов в устройствах магноники и спинтроники. Как показывают теоретические и экспериментальные исследования последних лет, движущиеся магнитные скирмионы обнаруживают нетривиальные физические свойства. Одним из динамических проявлений сложной микромагнитной структуры скирмионного состояния является асимметрия мод спиновых возбуждений, вращающихся в направлениях по и против часовой стрелки ( $\mathrm{CW}$ и $\mathrm{CCW}$ моды). Различия частот $\mathrm{CW}$ и CCW мод были определены на основе численных расчетов в 2012 г. [11], однако интерпретация спектров магнитных скирмионов, наблюдаемых экспериментально [12-15], а также рассчитанных методами микромагнитной симуляции [17-21], до сих пор является предметом активных научных дискуссий. Авторы работы [14] связывают асимметрию мод с гиротропными осцилляциями скирмиона, в экспериментах $[15,16]$ обнаружены две низкочастотные моды, исследования [12,13,17-21] указывают на наличие одной гиротропной моды в спектре возбуждений магнитных скирмионов.

Отметим другие динамические проявления структуры скирмиона: скирмионный эффект Холла (отклонение направления движения скирмиона от направления управляющего электрического тока) [22] и топологический эффект Холла (влияние создаваемого скирмионом неоднородного калибровочного магнитного поля и как следствие, электрического поля на его транспортные свойства) [23,24], которые пока не нашли полного теоретического объяснения.

В данной работе рассмотрены вопросы, связанные с возбуждениями скирмионного состояния в круглых наноточках. Рассчитаны спектры возбуждений скирмионов в изолированной круглой наноточке, проведена классификация спектра по высокочастотным модам различной пространственной симметрии и низкочастотным модам скирмионов с фиксированной фазой (скирмионов Блоха и Нееля). На основе проведенных расчетов выделены основные различия между гиротропной частотой и частотами спиновых волн изолированных скирмионов. Проанализирован спектр возбуждений линейной цепочки скирмионов, найдены характерные различия частот ферромагнитного резонанса, дисперсии и групповой скорости взаимодействующих блоховских и неелевских скирмионов.

\section{2. Постановка задачи и основные уравнения}

Исследование динамики скирмонных распределений намагниченности проводится в рамках двух подходов: непосредственного анализа уравнения Ландау-Лифшица и решения уравнения Тиля, которое является частным случаем уравнения Ландау-Лифшица, справедливое при солитонноподобной структуре рассматриваемого решения.

Для расчета динамических характеристик скирмионов удобно воспользоваться приближенной функцией, т.е.
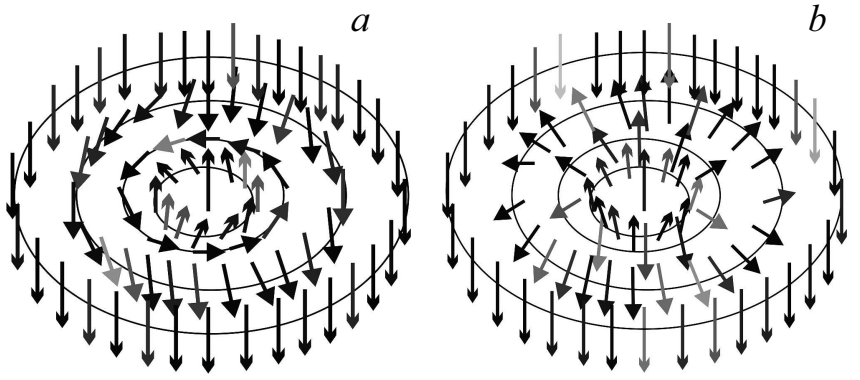

Pис. 1. Структура магнитного скирмиона: $a)$ скирмион Блоха (разворот намагниченности осуществляется в плоскости, перпендикулярной радиальному направлению), b) скирмион Нееля (разворот намагниченности происходит вдоль радиального направления).

использовать математический анзац, который позволяет описать структуру скирмионного состояния. Достаточно хорошим приближением является аналитическая функция Белявина-Полякова $f(z), z=x+i y$, полученная в 1975 г. при расчете метастабильных состояний изотропных 2D-магнетиков [25]. Последующие исследования, проведенные в работах [26,27], показали, что данный анзац является адекватным приближением и для описания вихревых магнитных состояний в изотропных магнетиках со значительным магнитостатическим взаимодействием.

Компоненты единичного вектора намагниченности $\mathbf{m}=\mathbf{M} / M_{s}$ можно выразить через функцию $f(z)$ следующим образом:

$$
m_{x}+i m_{y}=\frac{2 f(z)}{1+|f(z)|^{2}}, \quad m_{z}=\frac{1-|f(z)|^{2}}{1+|f(z)|^{2}} .
$$

Мы используем частный случай решения БелявинаПолякова (БП анзац) $f(z)=e^{i \Phi_{0}}(z-s) / c$, соответствующий одному скирмиону, центрированному в точке $s$, здесь $c=R_{c} / c-$ приведенный радиус магнитного скирмиона, $\Phi_{0}-$ угол, определяющий фазу скирмиона или характер разворота намагниченности в скирмионе, безразмерный параметр $\mathbf{s}=\mathbf{X} / R$ характеризует смещение центра скирмиона из положения равновесия в центре наноточки $(z=0)$, обусловленное действием внешних факторов, $R$ - радиус наноточки.

Мы предполагаем жесткую структуру динамического скирмионного состояния, т.е. учитываем только низкочастотные гиротропные моды и не рассматриваем вклад всех высокочастотных спиновых волн. В рамках такого подхода использование функции Белявина-Полякова для описания магнитных скирмионов с азимутальным и радиальным разворотом векторов намагниченности, где характер разворота определяется значением угла $\Phi_{0}$, является правомерным. Скирмионы с $\Phi_{0}=C \pi / 2$, где $C= \pm 1$ - параметр, определяющий киральность скирмиона, и $\Phi_{0}=0, \pi$ классифицируются как блоховские и неелевские скирмионы (рис. 1). 
Для расчета спектра возбуждений нам потребуется энергия магнетика, в которой мы будем учитывать энергию изотропного обмена, энергию магнитной анизотропии, энергию магнитостатики, энергию ДзялошинскогоМория, акцентируя внимание на скирмионах, стабилизируемых взаимодействием Дзялошинского-Мория, а также условиях, необходимых для реализации скирмионов при отсутствии взаимодействия Дзялошинского-Мория. Плотность энергии магнетика имеет вид

$$
F=A(\nabla \mathbf{m})^{2}+w_{D}-K m_{z}^{2}-\frac{1}{2} M_{s} \mathbf{m} \cdot \mathbf{H}_{m},
$$

где $A-$ константа обменного взаимодействия, $K>0-$ константа одноосной магнитной анизотропии, $\mathbf{m}=\mathbf{M} / M_{s}-$ единичный вектор намагниченности, $\mathbf{M}_{s}$ - намагниченность насыщения, $\mathbf{H}_{m}-$ магнитостатическое поле, $w_{D}$ - энергия Дзялошинского-Мория, представленная инвариантом Лифшица $w_{D} \sim \sum_{k=1}^{3}\left(m_{i} \frac{\partial m_{j}}{\partial x_{k}}-m_{j} \frac{\partial m_{i}}{\partial x_{k}}\right)$. Конкретный вид инварианта Лифшица, слагаемого линейного по пространственным производным намагниченности, определяется симметрией рассматриваемой системы. Энергия ДзялошинскогоМория имеет вид $w_{D}=D(\mathbf{m} \cdot \operatorname{rot} \mathbf{m})$ для кубических магнитных кристаллов симметрии В20 (классов симметрии $T, O)$ и $w_{D}^{*}=D\left[m_{z}(\nabla \cdot \mathbf{m})-(\mathbf{m} \cdot \nabla) m_{z}\right]$ для магнитных пленок и слоев вида $\mathrm{Co} / \mathrm{Pt}$, магнетиков классов симметрии $C_{n v}, C_{n}, D_{n}, D_{2 d}, S_{4}$ [28].

Рассмотрим скирмионы в системах ограниченной геометрии, а именно скирмионы в наноточках, учитывая взаимодействие Дзялошинского-Мория в качестве одного из факторов, приводящих к стабилизации скирмионного состояния. Для расчета спектров магнитных возбуждений, формирующихся на фоне скирмиона, используем два подхода: низкочастотные гиротропные моды будут исследованы на основе решения уравнения Тиля. Решение задачи о высокочастотных колебаниях намагниченности, спиновых волнах, будет проведено на основе приближенного решения уравнения ЛандауЛифшица.

\section{3. Гиротропные моды магнитных скирмионов}

Для расчета низкочастотной динамики магнитных скирмионов, связанной с гиротропными колебаниями намагниченности, используем уравнение Тиля, которое в общем случае имеет следующий вид

$$
\mathbf{G} \times \dot{\mathbf{X}}-\nabla_{X} W+\hat{D} \dot{\mathbf{X}}=0,
$$

где $\mathbf{G}=G_{z} \mathbf{z}, C_{z}=-p|G|$ - проекция гиротропного вектора на ось $O z, \mathbf{z}$ - радиус-вектор, направленный по нормали к поверхности наноточки вдоль оси $O z, \boldsymbol{\rho}-$ радиус-вектор в плоскости наноточки, $W(\mathbf{X})=L \int d^{2} \boldsymbol{\rho} w(\mathbf{X})$ полная энергия магнетика, $p= \pm 1-$ поляризация скирмиона, $\hat{D}-$ параметр затухания [29]. Величина гиротропного вектора скирмиона, расположенного в центре наноточки $(\mathbf{X}=0)$, пропорциональна топологическому заряду [30] и может быть рассчитана по формуле

$$
G_{z}=\frac{M_{s} L}{\gamma} \int d^{2} \boldsymbol{\rho} \mathbf{m} \cdot\left[\partial_{x} \mathbf{m} \times \partial_{y} \mathbf{m}\right] .
$$

При малых смещениях ядра скирмиона относительно положения равновесия, характеризуемого параметром $\mathbf{s}=\mathbf{X} / R, s=|\mathbf{s}| \ll 1$, полную энергию магнетика можно разложить в ряд по параметру $s$

$$
W(s)=W(0)+\kappa|s|^{2} / 2,
$$

где $W(0)$ - это энергия центрированного скирмиона, $\kappa$ - коэффициент при малом параметре $|s|^{2}$, который является коэффициентом жесткости. В случае ультратонких пленок $(L \sim 1 \mathrm{~nm}), \kappa(c)$ имеет вид (см. работу [31])

$$
\begin{aligned}
\kappa(c)= & -16 \pi M_{s}^{2} L \frac{c^{2}}{\left(1+c^{2}\right)^{3}} \\
& \times\left[\pi Q\left(1-c^{2}\right)+\left(\frac{L_{e}}{R}\right)^{2}\left(1+\frac{1}{2} \frac{R}{L_{e}} d c\right)\right],
\end{aligned}
$$

где $Q=K / 2 \pi M_{s}^{2}-$ фактор качества, $d=D L_{e} C / A$ $(d=-|d|<0$ для скирмионов с наименьшей энергией, $D C<0), L_{e}=\sqrt{2 A} / M_{s}$.

Гиротропная частота находится на основе решения уравнения Тиля (3) и для изолированного скирмиона в наноточках в ультратонких пленках определяется уравнением

$$
\begin{aligned}
\omega_{G}= & \omega_{M} \frac{c^{2}}{\left(1+c^{2}\right)^{2}} \\
& \times\left[(1-Q)\left(1-c^{2}\right)-\frac{1}{\pi}\left(\frac{L_{e}}{R}\right)^{2}\left(1+\frac{1}{2} \frac{R}{L_{e}} d c\right)\right],
\end{aligned}
$$

где $\omega_{M}=\gamma 4 \pi M_{s}, d<0$. График зависимости гиротропной частоты от радиуса наноточки при разных значениях константы Дзялошинского-Мория показан на рис. 2.

В уравнении (6) при определенных сочетаниях параметров системы коэффициент жесткости может иметь отрицательный знак, что, как следует из формулы (7), приводит к неустойчивости скирмионного состояния (относительно малых отклонений от положения равновесия, определяемых параметром $s$ ). Такая ситуация возникает в системах с малым значением константы Дзялошинского-Мория, в этом случае стабилизация скирмиона определяется конкуренцией между магнитостатической энергией и энергией магнитной анизотропии. Вопрос о стабилизации скирмионного состояния в наноточке при отсутствии взаимодействия ДзялошинскогоМория рассмотрен в работе [32]. В работе [32] было показано, что скирмион является устойчивым магнитным состоянием наноточки при комнатных температуpax в отсутствии магнитного поля и взаимодействия Дзялошинского-Мория в определенном диапазоне изменения полей магнитной анизотропии $(Q<1)$. 


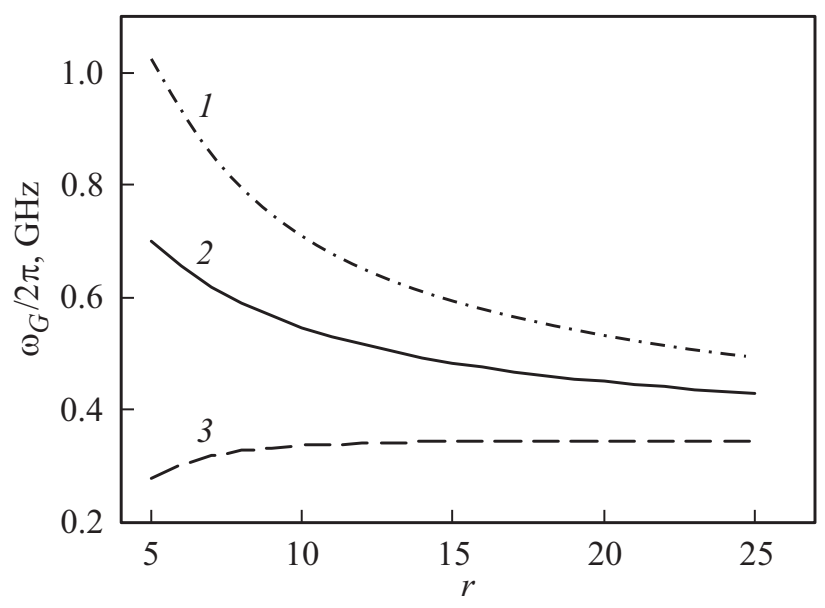

Рис. 2. График зависимости гиротропной частоты скирмиона Блоха, стабилизируемого в круглой магнитной наноточке, от приведенного радиуса наноточки $r=R / L_{e}$, кривая $1-|d|=4.90$, кривая $2-|d|=2.90$, кривая $3-$ $|d|=0.29\left(|D|=0.3 \mathrm{erg} / \mathrm{cm}^{2}\right), c=0.6, Q=0.94\left(M_{s}=1290 \mathrm{G}\right.$, $\left.A=1.5 \cdot 10^{-6} \mathrm{erg} / \mathrm{cm}, K=9.8 \cdot 10^{6} \mathrm{erg} / \mathrm{cm}^{3}, L_{e}=14 \mathrm{~nm}, R=50 \mathrm{~nm}\right)$.

Магнитостатическая энергия играет существенную роль при увеличении толщины наноточки $(L>1 \mathrm{~nm})$, а также при наличии нескольких скирмионов в системе, находящихся на расстояниях магнитостатической длины вплоть до $100 \mathrm{~nm}$. Наличие магнитостатического взаимодействия влияет на устойчивость скирмионных состояний, а также на спектры низкочастотных возбуждений скирмионов. Магнитостатическая энергия определяется формулой

$$
W_{m}(\mathbf{s})=\frac{1}{2} M_{s}^{2} \int d S \int d S^{\prime} \frac{\rho_{n}(\mathbf{r}, \mathbf{s}) \rho_{n}\left(\mathbf{r}^{\prime}, \mathbf{s}\right)}{\left|\mathbf{r}-\mathbf{r}^{\prime}\right|},
$$

где $\mathbf{r}$ - радиус-вектор, связанный с вектором намагниченности, $\rho_{n}(\mathbf{r}, \mathbf{s})$ - плотность „магнитных зарядов“, обусловленных локальным полем неоднородного распределения намагниченности в скирмионе. Различают объемные и поверхностные ,магнитные заряды“ $\rho_{\text {vol }}(\mathbf{r}, \mathbf{s})=-M_{s} \operatorname{div} \mathbf{m}$ и $\rho_{\text {surf }}(\mathbf{r}, \mathbf{s})=M_{s}(\mathbf{m} \cdot \mathbf{n})$. В случае центрированных блоховских скирмионов в магнитных наноточках поверхностные „магнитные заряды“ имеют одну составляющую $m_{z}=(\mathbf{m} \cdot \hat{\mathbf{z}})$ (боковые и объемные „магнитные заряды“ в этом случае отсутствуют). В случае неелевских скирмионов в наноточках присутствуют объемные „магнитные заряды“, а поверхностные заряды имеют две составляющие $m_{\rho}=(\mathbf{m} \cdot \hat{\boldsymbol{\rho}}), m_{z}=(\mathbf{m} \cdot \hat{\mathbf{z}})$. Как было показано в работе [33], учет магнитостатической энергии приводит к перенормировке частоты гиротропной моды изолированного скирмиона,

$$
\omega_{G}^{\prime}=\omega_{G}+\Delta \omega_{G}(c, \beta),
$$

где $\omega_{G}$ определяется формулой (7). В случае круглых магнитных наноточек в ультратонких пленках $(L \sim 1 \mathrm{~nm})$ гиротропная частота $\omega_{G}$ имеет одинаковый вид для скирмионов Блоха и Нееля. А в случае магнитных наноточек в пленках с толщиной $L>1 \mathrm{~nm}$ при увеличении толщины появляется дополнительный вклад в частоту $\Delta \omega_{G}(c, \beta)$, различный для блоховских и неелевских скирмионов из-за разного вклада динамических объемных и боковых поверхностных „магнитных зарядов“" (см. работу [33]).

В системе взаимодействующих скирмионов в разложении свободной энергии $W=\frac{1}{2} \sum_{n} \kappa_{n} s_{n} \overline{s_{n}}$ $+\frac{1}{2} \sum_{n, n^{\prime}} W_{\text {int }}\left(n, n^{\prime}\right)$ необходимо учитывать вклад, обусловленный энергией взаимодействия $W_{\text {int }}$, здесь $n-$ порядковый номер скирмиона в массиве скирмионов. В случае скирмионов, удаленных друг от друга на расстояние порядка магнитостатической длины, например, в линейной цепочке наноточек, энергия взаимодействия между скирмионами $W_{\text {int }}=\iint \frac{\rho\left(\mathbf{r}_{n}\right) \rho\left(\mathbf{r}_{n^{\prime}}\right)}{\left|\mathbf{r}_{n}-\mathbf{r}_{n^{\prime}}-\mathbf{R}_{n n^{\prime}}\right|} d V d V^{\prime}$ может быть представлена в виде разложения по мультипольным моментам $Q_{l}^{m}=\sqrt{4 \pi /(2 l+1)} \int d V \rho(\mathbf{r}) r^{l} Y_{l}^{m}(\mathbf{r})$, где $l-$ номер мультипольного момента, $m=-l, 0,+l, Y_{l}^{m}(\mathbf{r})-$ нормированные сферические функции [34]. Отличительной особенностью скирмионных состояний является значительный вклад в распределение намагниченности в скирмионе $m_{z}$ компоненты намагниченности, что приводит к появлению квадрупольных моментов $Q_{2}^{m}(n)$ и дополнительному вкладу в дипольный момент скирмиона $Q_{1}^{0}(n)$. Хотя диполь-дипольное взаимодействие является основной составляющей магнитостатического взаимодействия, учет вклада квадрупольных моментов и моментов более высокого порядка приводит к заметным изменениям спектров частот. В работе [35] показано, что частота коллективных колебаний цепочки скирмионов определяется выражением

$$
\begin{gathered}
\omega_{k}^{2}=\left(\omega_{G}+\Delta \omega-a_{k}-\left|b_{k}\right|\right)\left(\omega_{G}+\Delta \omega-a_{k}+\left|b_{k}\right|\right), \quad(10) \\
\Delta \omega=-\frac{1}{4} \omega_{M} \frac{\beta}{d^{3}} \mu_{z}^{0} \sigma^{d}(0) \frac{c^{2}}{1+c^{2}}, \omega_{d}=-\omega_{M} \frac{\beta}{8 d^{3}} \frac{c^{2}}{1+c^{2}}, \\
\omega_{q}=\omega_{M} \frac{\beta}{d^{4}} c^{3}\left(\ln \left(1+\frac{1}{c^{2}}\right)-\frac{1}{1+c^{2}}\right), \\
a_{k}=-\omega_{d} \sigma_{k}^{d}+\omega_{q} \sigma_{k}^{q} \sin \Phi_{0}, \\
\left|b_{k}\right|=\sqrt{\frac{9}{4} \omega_{d}^{2}\left(\sigma_{k}^{d}\right)^{2}+\omega_{q}^{2}\left(\sigma_{k}^{q}\right)^{2}}, \\
\sigma_{k}^{d}=2 \sum_{n=1}^{\infty} \frac{\cos (n k)}{n^{3}}, \quad \sigma_{k}^{q}=2 \sum_{n=1}^{\infty} \frac{\cos (n k)}{n^{4}}, \quad \beta=\frac{L}{R} .
\end{gathered}
$$

Спектр возбуждений массива скирмионов имеет зонную структуру, причем ширина и положение зоны, как следует из формулы (10), зависит от фазы скирмиона $\Phi_{0}$. Подобная зависимость отсутствовала в формулах (7), (9) для гиротропной моды изолированного скирмиона. Это говорит о том, что внутренняя структура скирмионов проявляется при динамическом взаимодействии скирмионов. Рассчитанная по формуле (10) разница 
частот ферромагнитного резонанса блоховских и неелевских скирмионов имеет порядок $0.1 \mathrm{GHz}$ для материалов с параметрами [14], что говорит о возможности экспериментальной регистрации структуры магнитных скирмионов по динамическому отклику, в частности методом широкозонного ферромагнитного резонанса.

\section{4. Спиновые волны в спектре колебаний изолированного магнитного скирмиона в наноточке}

Рассмотрим особенности спектров спиновых волн, возбуждаемых на фоне скирмионного состояния в изолированной наноточке. Для этого перейдем к угловым переменным, определяющим равновесное положение вектора намагниченности $\mathbf{m}=$ $=(\sin \Theta \cos \Phi, \sin \Theta \sin \Phi, \cos \Theta)$, где $\Theta$ и $\Phi-$ полярный и азимутальный углы в сферической системе координат, связанной с вектором $\mathbf{m}$, и к цилиндрическим координатам, используемым для описания радиусвектора $\mathbf{r}(\boldsymbol{\rho}, z)$, где $\boldsymbol{\rho}$ - радиус-вектор в плоскости наноточки, $\rho, \varphi-$ полярные координаты в плоскости наноточки, $z$ - координата вдоль высоты наноточки.

Уравнение Ландау-Лифшица $\dot{M}=-\gamma\left[\mathbf{M} \times \mathbf{H}_{\mathrm{eff}}\right]$ $+\frac{\alpha}{M_{0}}[\mathbf{M} \times \dot{\mathbf{M}}], \mathbf{H}_{\mathrm{eff}}=-\frac{\delta F}{\delta \mathbf{M}}$ в угловых переменных $(\Theta, \Phi)$ имеет вид

$$
\begin{aligned}
\frac{M_{s}}{2 \gamma} \sin \Theta \dot{\Theta}= & A\left(\left(\nabla^{2} \Phi\right) \sin ^{2} \Theta+(\nabla \Theta \cdot \nabla \Phi) \sin 2 \Theta\right) \\
& -D(\nabla \Theta \cdot \mathbf{m}) \sin \Theta \\
\frac{M_{s}}{2 \gamma} \sin \Theta \dot{\Phi}= & {\left[K+A(\nabla \Phi)^{2}\right] \sin \Theta \cos \Theta } \\
& -A \nabla^{2} \Theta-D(\nabla \Phi \cdot \mathbf{m}) \sin \Theta .
\end{aligned}
$$

Мы пренебрегаем затуханием в системе, полагая $\alpha=0$, что является вполне оправданным, так как параметр затухания в рассматриваемых системах имеет величину порядка 0.01. Предполагая возбуждения скирмионного состояния малыми, линеаризуем уравнения (11), (12). Для этого перейдем к локальной системе координат, связанной с равновесной намагниченностью $\mathbf{m}_{0}\left(\mathbf{m}_{0} \cdot \delta \mathbf{m}=0\right)$, и используем замену $\mathbf{m}(\mathbf{r}, t)=\mathbf{m}_{0}(\mathbf{r})+\delta \mathbf{m}(\mathbf{r}, t)$. В выбранной локальной системе координат ось $O z^{\prime}$ совпадает с направлением равновесной намагниченности, динамическая намагниченность $\delta \mathbf{m}$ вращается в плоскости $x^{\prime} O y^{\prime}$. В данном случае отклонения намагниченности от равновесного положения, выраженные в угловых переменных, примут вид

$$
\begin{gathered}
\Theta(\boldsymbol{\rho}, t)=\Theta_{0}(\rho)+\vartheta(\boldsymbol{\rho}, t), \quad \Phi(\boldsymbol{\rho}, t)=\Phi_{0}(\boldsymbol{\rho})+\psi(\boldsymbol{\rho}, t), \\
\vartheta(\boldsymbol{\rho}, t)=a_{n m}(\rho) \cos (m \varphi-\omega t), \\
\mu(\boldsymbol{\rho}, t)=-\sin \Theta_{0} \psi(\boldsymbol{\rho}, t)=b_{n m}(\rho) \sin (m \varphi-\omega t),
\end{gathered}
$$

где функции $a_{n m}$ и $b_{n m}$ определяют радиальные профили магнонных мод, $n$ и $m$ - номера радиальных и ази- мутальных мод, $\omega-$ собственная частота колебаний магнитного скирмиона.

Будем считать, что профиль скирмиона определяется пробной функцией, описывающей распределение намагниченности в радиальной доменной границе $\operatorname{tg} \frac{\Theta_{0}(\rho)}{2}=\exp \frac{\rho-R_{c}}{\Delta} \quad$ (здесь $R_{c}-$ координата центра доменной границы или радиус скирмиона, $\Delta=L_{e} / \sqrt{4 \pi(Q-1)}$ - ширина доменной границы). Таким образом, мы допускаем, что при $\Theta_{0}(0)=0$ скирмион имеет полярность $p=\cos \Theta_{0}(0)=+1$. Отметим, что линеаризованные уравнения динамики, которые получаются после подстановки уравнений (13), (14) в систему уравнений (11), (12), не зависят от фазы скирмиона, а именно являются общими для изолированных скирмионов блоховского и неелевского типа. Мы также предполагаем ступенчатую зависимость компоненты $m_{z}$ от радиальной координаты, характерную для ультратонких радиальных доменных границ $\left(R_{c} \gg \Delta\right)$. Подробное решение данной задачи представлено в работе [36], здесь мы акцентируем внимание на основных зависимостях, которые позволяют выявить особенности высокочастотного спинволнового спектра скирмионного состояния намагниченности в цилиндрической наноточке. Частоты высокочастотных спиновых мод скирмионного состояния в наноточках в ультратонких пленках в рамках обменного приближения определяются уравнением

$$
\frac{\omega_{n m}}{\omega_{M}}=Q-1+\frac{1}{4 \pi}\left(\frac{L_{e}}{R_{c}}\right)^{2} x_{n m}^{2},
$$

где $L_{e}=\sqrt{2 A} / M_{s} \approx 15-20 \mathrm{~nm}$ - обменная длина рассматриваемого магнитного материала, $x_{n, m}-$ корни секулярного уравнения [36], полученного из граничных условий для радиальных профилей спинволновых мод $a_{n m}(\rho), b_{n m}(\rho)$ (условия непрерывности распределения намагниченности на границе цилиндрического магнитного домена, соответствующей радиусу скирмиона $R_{c}$, и условия сильного пиннинга, т.е. закрепления спинов на границе наноточки).

На рис. 3 показаны зависимости собственных частот линеаризованных уравнений Ландау-Лифшица (11), (12) от радиуса скирмиона в единицах радиуса наноточки $\left(R_{c} / R\right)$. Магнитные параметры, использованные для расчета, соответствуют экспериментальной работе [14], в которой исследовалась динамика магнитных скирмионов в тонких $\mathrm{Co} / \mathrm{Pt}$ наноточках. Отметим, что в рамках приближения ультратонкой доменной границы частоты спиновых волн явным образом не зависят от константы Дзялошинского-Мория $D$. Однако такая зависимость явно присутствует в зависимости равновесного радиуса скирмиона $R_{c}$ от параметра $D$. Увеличение толщины наноточки приводит к стабилизации уже существующего скирмиона Блоха. В случае неелевских скирмионов происходит постепенный переход от скирмиона с конфигурацией Нееля к скирмиону с конфигурацией Блоха, обусловленный увеличением магнитостатической энергии, связанной с объемными и боковыми поверхностными „магнитными зарядами“. 


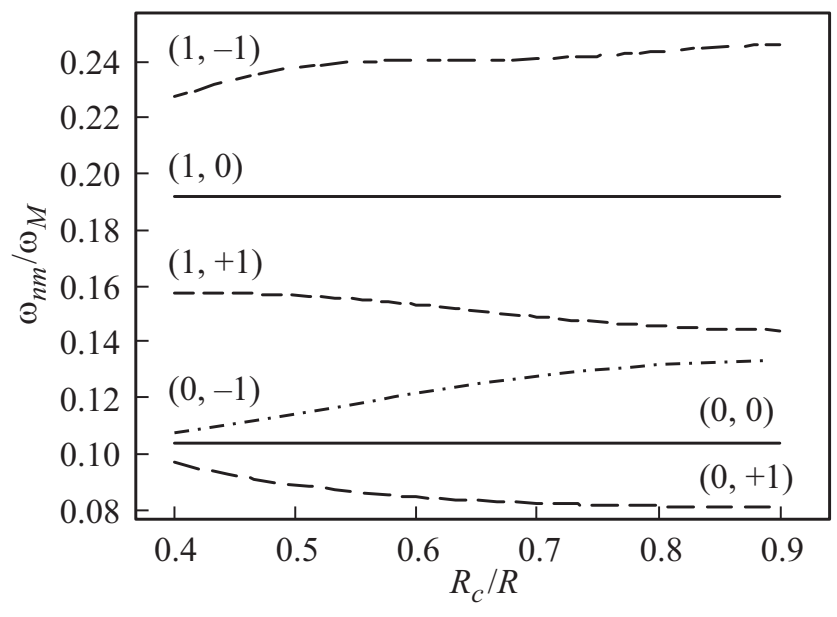

Рис. 3. График зависимости приведенных частот спиновых волн $\omega_{n m} / \omega_{M}$, возбуждаемых на фоне скирмиона в круглой магнитной наноточке радиуса $100 \mathrm{~nm}$ в зависимости от радиуса скирмиона $R_{c} / R . M_{s}=1000 \mathrm{G}, A=1.6 \cdot 10^{-6} \mathrm{erg} / \mathrm{cm}^{2}$, $K=6.7 \cdot 10^{6} \mathrm{erg} / \mathrm{cm}^{3}, L_{e}=18 \mathrm{~nm}, \omega_{M} / 2 \pi=37.1 \mathrm{GHz}, R=100 \mathrm{~nm}$.

Характерная асимметрия собственных частот спиновых мод, различающихся знаком азимутального индекса $m$, который определяет азимутальные моды колебаний (рис. 3), говорит о том, что спиновые волны, соответствующие вращениям динамической намагниченности вокруг центра наноточки по и против часовой стрелки, различны. Это непосредствнно связано с профилем скирмиона $m_{z}(\rho)$ или с наличием ненулевого топологического заряда, пропорционального гировектору, определенного формулой (4). Секулярное уравнение несимметрично относительно замены $m \rightarrow-m$. Оно имеет более сложную комбинационную симметрию, связанную с одновременной заменой $m \rightarrow-m$ и $p \rightarrow-p$. Азимутальные спиновые волны с индексами $m=+1 /-1$ имеют качественные различия радиальных профилей для любых $n$ (профили $a_{n m}(\rho), b_{n m}(\rho)$ пропорциональны функциям Бесселя 1-го рода $\left.J_{m-1}\left(x_{n m} \rho / c R\right)\right)$. При этом профили спиновых волн с индексами $\pm|m|$ при $|m|>1$ качественно идентичны. Положительная полярность $p=+1$ скирмиона соответствует гиротропному вращению центра скирмиона против часовой стрелки $(\mathrm{CCW})$, которое сопровождается азимутальной спиновой волной с индексом $m=+1$, также вращающейся против часовой стрелки. Спиновая волна с $m=+1$ имеет высокую амплитуду, локализованную во внутреннем домене наноточки, и классифицируется как поддержанная спинволновая мода $\sim J_{0}\left(x_{n 1} \rho / c R\right)$. Спиновая волна с индексом $m=-1(\mathrm{CW})$ вращается в противоположном гиротропному вращению направлении (по часовой стрелке). Как следствие ее амплитуда, локализованная во внутреннем домене, понижается вплоть до исчезновения, эта мода классифицируется как подавленная спинволновая мода $\sim J_{2}\left(x_{n,-1} \rho / c R\right)$. Остальные моды спиновых волн с азимутальными индексами $m=0$ и $|m|>1$ также подавляются в окрестности центра наноточки, так как их симметрия не соответствует симметрии гиротропной моды, определяемой индексами $m=+1 /-1$.

\section{5. Заключение}

В данной работе рассмотрены основные аспекты динамики магнитных скирмионов в нанототочках, включая расчет спектров спиновых волн и гиротропных мод изолированного скирмиона и системы взаимодействующих скирмионов на примере 1D массива. Проведена классификация мод возбуждений скирмионного состояния. Показано, что при возбуждении скирмионов в изолированной наноточке возбуждается единственная низкочастотная гиротропная мода, основными характеристиками которой являются: самая низкая частота в спектре возбуждений и локализация моды в центральной области наноточки. Т. е. гиротропная мода связана с осцилляциями центральной части (ядра) скирмиона. Определение частоты гиротропной моды имеет значение, в частности, для процессов динамического переключения полярности скирмионного состояния, которое осуществляется через гиротропную моду. Исследования высокочастотного спектра возбуждений скирмионов в наноточке показали, что азимутальные спиновые волны, распространяющиеся на фоне скирмиона, характеризуются асимметрией по отношению к вращению моды: частоты и профили азимутальных мод, вращающихся по и против часовой стрелки $(\mathrm{CW}$ и $\mathrm{CCW})$, различны. Отличительной особенностью динамики изолированных скирмионов в круглых наноточках является то, что спектр возбуждений, как низкочастотный (гиротропное вращение ядра скирмиона), так и высокочастотный (спиновые волны), является идентичным для скирмионов Блоха и Нееля. Внутренняя структура скирмионного состояния проявляется при учете взаимодействия между скирмионами. Исследование спектра линейной цепочки наноточек с основным скирмионным состоянием показало существенное различие динамических характеристик (частот ферромагнитного резонанса (ФМР), закона дисперсии, групповой скорости, ширины магнонной зоны) для скирмионов блоховского и неелевского типов, что позволяет предложить метод экспериментального обнаружения распределения спинов в скирмионе по динамическому отклику скирмионной системы, а именно по частоте ФМР, которая может быть зарегистрирована методом широкозонного ферромагнитного резонанса.

\section{Список литературы}

[1] S. Mühlbauer, B. Binz, F. Jonietz, C. Pfleiderer, A. Rosch, A. Neubauer, R. Georgii, P. Boeni. Science 323, 915 (2009).

[2] X.-Z. Yu, Y. Onoze, N. Kanazawa, J.H. Park, J.H. Han, Y. Matsui, N. Nagaosa, Y. Tokura. Nature 65, 901 (2010).

[3] S. Seki, X.Z. Yu, S. Ishiwata, Y. Tokura. Science 336, 198 (2012). 
[4] C. Moreau-Luchaire, N. Reyren, J. Sampaio, C.A.F. Vaz, N. Van Horne, K. Bouzehouane, K. Garcia, C. Deranlot, P. Warnicke, P. Wohlhẗer, J.-M. George, M. Weigand, J. Raabe, V. Cros, A. Fert. Nature Nanotechnol. 11, 444 (2016).

[5] O. Boulle, J. Vogel, H. yang, S. Pizzini, D.S. Chaves, A. Locatelli, T.O. Mentes, A. Sala, L.D. Buda-Prejbeanu, O. Klein, M. Belmeguenai, Y. Roussigne, A. Stashkevich, S.M. Cherif, L. Aballe, M. Foerster, M. Chshiev, S. Auffret, I.M. Miron, G. Gaudin. Nature Nanotechnol. 11, 449 (2016).

[6] S. Woo, K. Litzius, B. Krüger, M.Y. Im, M.Y. Caretta, K. Richter, M. Mann, A. Krone, R. Reeve, M. Weigand, P. Agrawal, P. Agrawal, P. Fischer, M. Kläui, G.S.D. Beach. Nature Mater. 15, 501 (2016).

[7] L. Sun, R.X. Cao, B.F. Miao, B. You, D. Wu, W. Zhang, A. Hu, H.F. Ding. Phys. Rev. Lett. 110, 167201 (2013).

[8] B.F. Miao, L. Sun, Y.W. Wu, X.D. Tao, X. Xiong, Y. Wen, R.X. Cao, P. Wang, D. Wu, Q.F. Zhan, B. You, J. Du, R.W. Li, H.F. Ding. Phys. Rev. B 90, 174411 (2014).

[9] M. Mruczkiewicz, P. Gruszecki, M. Zelent, M. Krawczyk. Phys. Rev. B 93, 174429 (2016).

[10] J. Kim, Y. Yang, Y.J. Cho, B. Kim, S.K. Kim. Sci. Rep. 7, 45185 (2017).

[11] M. Mochizuki, S. Seki. J. Phys.: Condens Matter 27, 503001 (2015).

[12] Y. Onose, Y. Okamura, S. Seki, S. Ishiwata, Y. Tokura. Phys. Rev. Lett. 109, 037603 (2012).

[13] Y. Okamura, F. Kagawa, M. Mochizuki, M. Kubota, S. Seki, S. Seki, S. Ishiwata, M. Kawasaki, Y. Onose, Y. Tokura. Nature Commun. 4, 2391 (2013).

[14] F. Buettner, C. Moutafis, M. Schneider, B. Krüger, C.M. Günther, J. Geilhufe, C. v. Korff Schmising, J. Mohanty, B. Pfau, S. Schaffert, A. Bisig, M. Foerster, T. Schulz, C.A.F. Vaz, J.H. Franken, H.J.M. Swagten, M. Kläui, S. Eisebitt. Nature. Phys. 11, 225-228 (2015).

[15] T. Schwarze, J. Waizner, M. Garst, A. Bauer, I. Stasinopoulos, H. Berger, C. Pfleiderer, D. Grundler. Nature Mater. 14, 478483 (2015).

[16] N. Ogawa, S. Seki, Y. Tokura. Sci. Rep. 5, 9552 (2015).

[17] S.Z. Lin, C.D. Batista, A. Saxena. Phys. Rev. B 89, 024415 (2014).

[18] S. Zhang, J. Wang, Q. Zheng, Q. Zhu, X. Liu, S. Chen, C. Jin, Q. Liu, C. Jia, D. Xue. New J. Phys. 17023061 (2015).

[19] F. Garcia-Sanchez, J. Sampaio, N. Reyren, V. Cros, J.V. Kim. New J. Phys. 18, 075011 (2016).

[20] M. Beg, M.A. Bisotti, D. Cores-Ortuno, W. Wang, R. Carey, M. Vousden, O. Novorka, C. Ciccarelli, C.S. Spencer, C.H. Marrows, H. Fangohr. Phys. Rev. B 95, 014433 (2017).

[21] M. Mruczkiewicz, M. Krawczyk, K.Y. Guslienko. Phys. Rev. B 95, 094414 (2017).

[22] W. Jiang, X. Zhang, G. Yu, W. Zhang, M.B. Jungfleisch, J.E. Pearson, O. Heinonen, K.L. Wang, Y. Zhou, A. Hoffmann, S.G.E. Velthuis. Nature Phys. 13, 162 (2017).

[23] A. Neubauer, C. Pfleiderer, B. Binz, A. Rosch, R. Ritz, P.G. Niklowitz, P. Böni. Phys. Rev. Lett. 102, 186602 (2009).

[24] T. Schulz, R. Ritz, A. Bauer, M. Halder, M. Wagner, C. Franz, C. Pfleidere. Nature Phys. 8, 301 (2012).

[25] А.А. Белавин, А.М. Поляков. Письма в ЖЭТФ 22, 503 (1975).
[26] Н.А. Усов, С.Е. Песчаный. ФММ 78, 13 (1994).

[27] K.L. Metlov, K.Y. Guslienko. J. Magn. Magn. Mater. 242-245, 1015 (2002).

[28] A.O. Leonov, T.L. Monchesky, N. Romming, A. Kubetzka, A.N. Bogdanov, R. Wiesendanger. New J.Phys. 18, 065003 (2016).

[29] K.Y. Guslienko, J. Nanosci. Nanotechnol. 8, 2745 (2008).

[30] K.Y. Guslienko. EPL 113, 67002 (2016).

[31] K.Y. Guslienko, Z.V. Gareeva. IEEE Magn. Lett. 8, 4100305 (2017).

[32] K.Y. Guslienko. IEEE Magn. Lett. 6, 4000104 (2015).

[33] K.Y. Guslienko, Z.V. Gareeva. J. Magn. Magn. Mater. 442, 176-182 (2017).

[34] O.V. Sukhostavets, J. Gonzalez, K.Y. Guslienko. Phys. Rev. B 87, 094402 (2013).

[35] Z.V. Gareeva, K.Y. Guslienko. J. Phys.Commun. (2017).

[36] Z.V. Gareeva, K.Y. Guslienko. Phys. Stat. Solidi (RRL) Rapid Res. Lett. 10, 227-232 (2016). 